\title{
HIPMap: A High-Throughput Imaging Method for Mapping Spatial Gene Positions
}

\author{
Sigal Shachar, Gianluca Pegoraro, and Tom Misteli \\ National Cancer Institute, National Institutes of Health, Bethesda, Maryland 20892 \\ Correspondence: mistelit@mail.nih.gov
}

\begin{abstract}
The three-dimensional organization of genes inside the cell nucleus affects their functions including DNA transcription, replication, and repair. A major goal in the field of nuclear architecture is to determine what cellular factors establish and maintain the position of individual genes. Here, we describe HIPMap, a high-throughput imaging and analysis pipeline for the mapping of endogenous gene loci within the 3D space of the nucleus. HIPMap can be used for a variety of applications including screening, mapping translocations, validating chromosome conformation capture data, probing DNA-protein interactions, and interrogation of the relationship of gene expression with localization.
\end{abstract}

A fundamental property of genomes is their complex higher-order organization (Misteli 2007). Genomes across species and phyla are arranged at multiple, hierarchical levels. The DNA, wrapped around proteinaceous nucleosomes, forms a chromatin fiber which in turn is locally compacted into topologically associated domains (Dixon et al. 2012; Sexton et al. 2012). These domains aggregate to give rise to chromosome subdomains and ultimately chromosome territories (Cavalli and Misteli 2013). The individual chromosomes, and consequently the genes they carry, are positioned in $3 \mathrm{D}$ space of the nucleus in a nonrandom fashion creating distinct spatial genome neighborhoods. The higher-order organization of the genome at all levels, including the spatial position in the nucleus, has been implicated in most genome functions and cellular processes (Misteli 2013). Although lowerlevel organization determines local regulatory effects such as nucleosome density, many gene regulatory functions appear to emerge from short-range chromatin interactions such as enhancer-promoter loops and longerrange effects such as clustering of multiple gene loci in 3D space (Dekker and Misteli 2015). The mechanisms that determine the higher-order spatial organization of genomes are largely unknown.

Although much of what we know about the lower-level organization of the genome at the scale of nucleosomes comes from traditional biochemical methods, insight into higher-order organization has come from two major approaches: imaging, mostly using fluorescence in situ hybridization (FISH) (Cremer and Cremer 2010), and cross-linking mapping, mostly using chromosome conformation capture (3C) methods (Dekker et al. 2013). Both approaches have advantages and limitations. The major strength of imaging methods is their ability to probe the location and physical proximity of multiple genome regions at the single-cell level and to derive spatial distance measurements, within the limits of the opti- cal resolution of the microscope. A shortcoming of imaging is its very low throughput, which typically allows only probing of a handful of genes or samples in a single experiment, thus precluding systematic genome-wide analysis. This limitation is powerfully overcome by $3 \mathrm{C}$ methods, which rely on chemical cross-linking of interactions throughout the genome, allowing systematic, genome-wide mapping of chromatin interactions in a single experiment (de Wit and de Laat 2012). However, $3 \mathrm{C}$-based methods are restricted to determining pairwise interactions and do not allow direct distance measurements or analysis of multiple, simultaneous interactions, such as gene clusters. In addition, their population-based nature, which uses data from millions of cells in aggregate, makes it a priori impossible to determine in how many cells in the population a particular interaction occurs or whether combinations of interactions occur simultaneously in an individual cell (Williamson et al. 2014). Early single-cell 3C methods are beginning to address some of these issues, albeit at the cost of reduced resolution $(\mathrm{Na}-$ gano et al. 2013). A significant weakness shared by both imaging-based and $3 \mathrm{C}$ genome mapping methods is their unsuitability for use in unbiased, large-scale screens due to the low throughput in the case of imaging and the technical complexity of $3 \mathrm{C}$ approaches, thus limiting their usefulness in unbiased discovery approaches.

An approach that overcomes some of the limitations of traditional imaging and $3 \mathrm{C}$ methods and bridges the gap between conventional imaging and C-technology is highthroughput imaging (HTI) (Roukos et al. 2010). This strategy consists of highly parallel processing and automated imaging of thousands of samples, automated image analysis, and statistical evaluation of localization patterns. HTI allows the analysis of a large number of cells in thousands of samples and combines the ability of imaging to generate single-cell data with the capacity of $3 \mathrm{C}$ methods to interrogate genome organization pat- 
terns at a large scale. In addition, the large-scale nature of HTI enables unbiased screening approaches (Conrad and Gerlich 2010).

We have recently developed an HTI-based pipeline for the mapping of genome locations and distances in 3D space (Burman et al. 2015a,b; Shachar et al. 2015). HTI positioning mapping (HIPMap) is a robust and versatile method to probe gene location and genome organization and we have shown its suitability in RNAi-based screens (Shachar et al. 2015). We describe here technical details and potential applications of HIPMap.

\section{HIPMap}

HIPMap was developed for the purpose of studying the position of a gene in the 3D space of the nucleus in a robust, quantitative, and high-throughput manner
(Shachar et al. 2015). HIPMap consists of an optimized pipeline that includes high-throughput FISH (hiFISH), automated image analysis, and subsequent statistical data analysis (Fig. 1).

\section{hiFISH}

The first step in the HIPMap protocol is hiFISH performed in 96- or 384-well plates using a fully automated liquid handling system. Fibroblasts are plated in 384-well imaging plates at $\sim 80 \%$ confluence and are grown for at least $12 \mathrm{~h}$ to adhere. For cells in suspension, wells are precoated with poly-L-lysine before plating. Cells are then transfected with siRNAs or expression constructs or treated with compounds depending on the desired experiment. A key feature for successful FISH on a large scale is to streamline the FISH protocol by reducing the number of

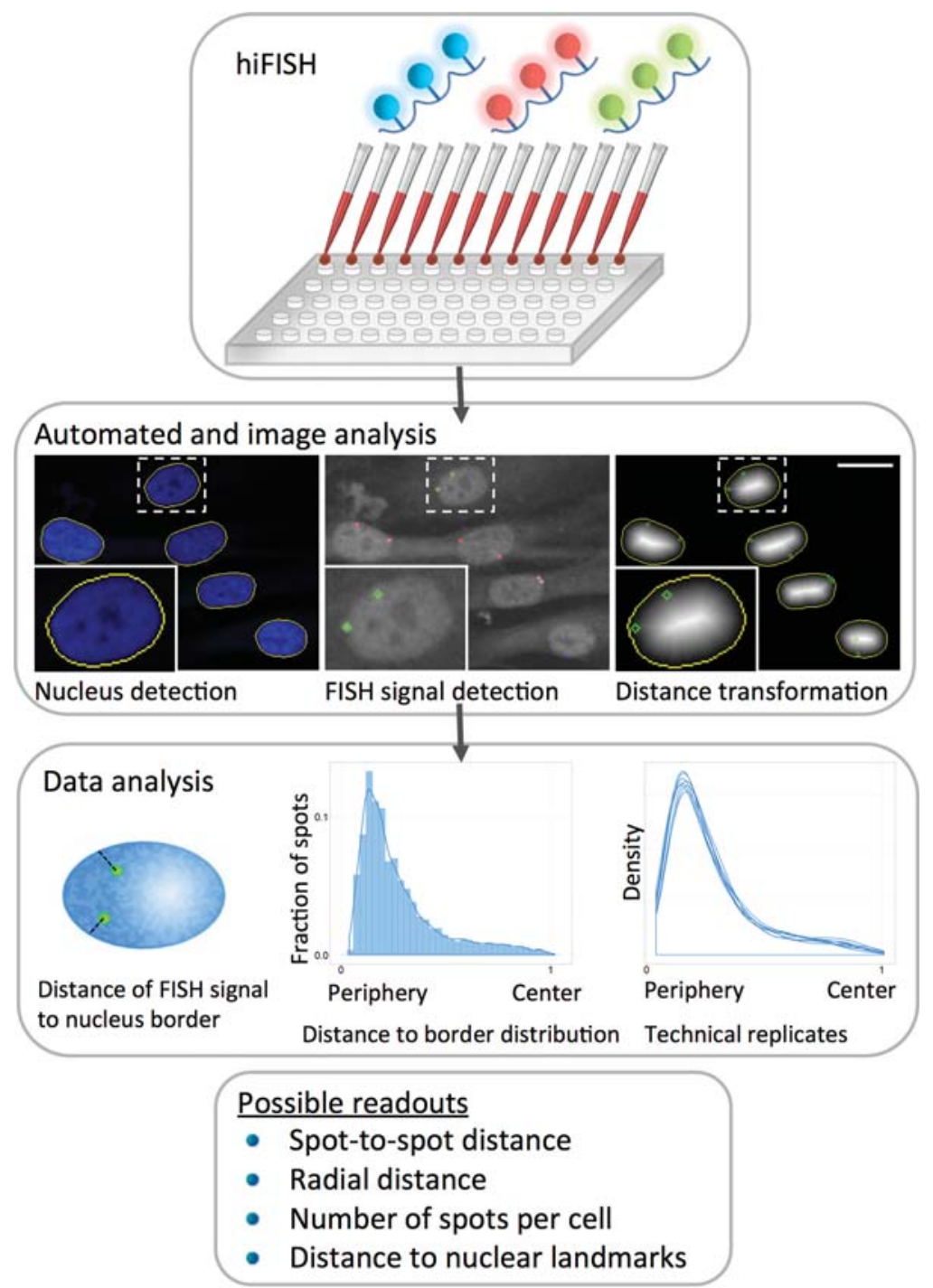

Figure 1. Outline of HIPMap pipeline. Cell plating and FISH (fluorescence in situ hybridization) protocol are performed in a 384-well format in a fully automated manner using fluorescently labeled FISH probes. Representative maximally projected images of $z$-stacks are analyzed to identify nuclei and detect FISH signals inside each nucleus. The normalized radial distance of each FISH spot from the nucleus border is measured and all distance measurements are combined and plotted as histograms or estimated density curves. 
steps. HiFISH probes are generated from bacterial artificial chromosomes (BACs) using a standard nick translation protocol with fluorescent nucleotides, thereby eliminating subsequent antibody detection steps as would be needed for biotin-labeled probes or alike. The protocol is also optimized to minimize the number of wash steps to avoid detachment of cells from the plate (Shachar et al. 2015).

\section{Automated Imaging}

In the next step, an automated confocal microscope is used to acquire images. In a standard application, samples are imaged in four channels (three probe channels plus DAPI) typically using the 405-, 488-, 561-, and 640-nm laser lines with a $40 \times$ water objective and $2 \times$ pixel binning. Typically, 60 fields are randomly sampled in a well and six $z$ planes (1- $\mu \mathrm{m}$ intervals) are acquired yielding 1000 to 3000 cells per well, depending on cell size and plating density. The average exposure and imaging time per well using these conditions is $\sim 20 \mathrm{~min}$ and the pixel size is $320 \mathrm{~nm}$. Exposure time varies according to cell type and density.

\section{Image Analysis}

Images are analyzed in a fully automated fashion to detect cell nuclei and FISH signals. The nucleus border is segmented based on the DAPI staining with high accuracy. FISH spots in all three channels are identified based on detection of local signal maxima in each probe channel. Radial distances from the nuclear edge are measured for every individual FISH signal in a nucleus. Each nucleus undergoes a radial distance transformation in which the border of the nucleus is set as 0 and the center of the nucleus is set as 1. FISH signals are superimposed onto the distance transformation image and their radial coordinates within each nucleus measured (Fig. 1). HIPMap can also measure spot-to-spot Euclidian distances by using the image $x$ and $y$ coordinates of each FISH spot. Spot-to-spot distances can be used to measure distances between alleles or genes or to nuclear landmarks. A detailed description of all image analysis steps is described in Roukos et al. (2013), Burman et al. (2015a), and Shachar et al. (2015).

\section{Data Analysis}

To determine the spatial position of a gene inside the nucleus, its distance from the border of the nucleus is measured in hundreds of cells in a population. Individual allele position measurements are combined and plotted as a radial position distribution (Fig. 1). This distribution provides a representation of the overall position of a gene.

For comparison of two distributions, data sets are transformed into cumulative distribution functions (Meaburn and Misteli 2008; Meaburn et al. 2009) and the Kolmogorov-Smirnov (KS) test (Perlman et al. 2004; Mitchison 2005; Smellie et al. 2006) is applied to determine whether the two distributions are different. The KS test is used because it is a nonparametric statistical test that is sensitive to local changes in the shape of the distributions rather than just variations in the mean or median.
To simplify the image analysis, all z-stacks are projected to generate 2D images, and spot coordinates and distances to the nucleus border are measured using only the $x$ and $y$ dimensions. To verify that such an analysis approximates the actual 3D distances and is reliable, we compared spot-to-spot distances in 3D with that in 2D for the same data set. We analyzed human skin fibroblasts and examined the distance between two endogenous loci that reside on two different chromosomes and are not known to interact ( $L A D F$ and COL1A1; Shachar et al. 2015). Minimal distances between each pair of spots per cell were calculated in projected images or in 3D using six $z$ planes 1 $\mu \mathrm{m}$ apart (Fig. 2A). The tested cells were treated with a nontargeting siRNA (small interfering RNA) or with an siRNA directed toward $\operatorname{Lamin} A / C$ and $\operatorname{LaminB} 1$ that was
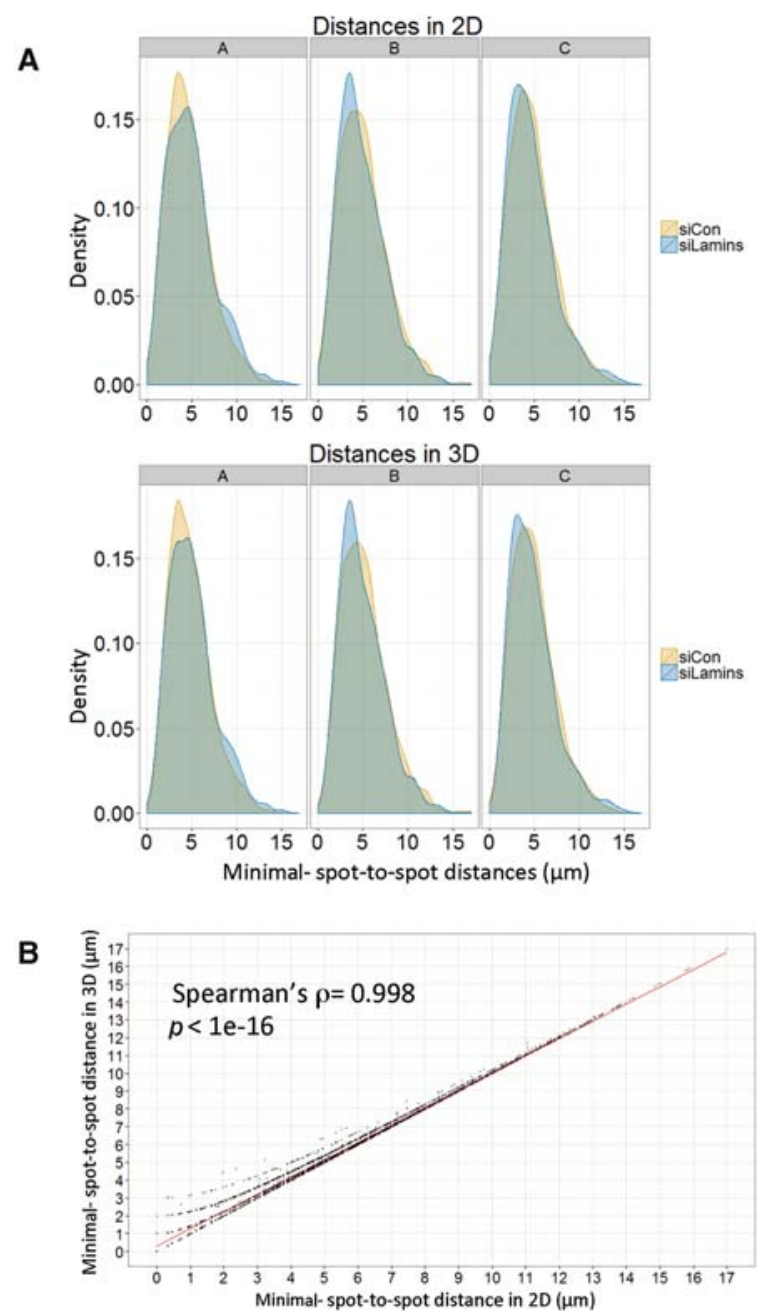

Figure 2. Distance measurement in $2 \mathrm{D}$ versus 3D. (A) Comparison of 2D with 3D analysis. Spot-to-spot distance distributions are similar between 2D and 3D analyses. Density curves of spotto-spot distances of $L A D F$ and COL1A1 in cells treated with nontargeting siRNA or knocked-down for LaminA / C and Lamin $B 1$ are measured in $2 \mathrm{D}$ in maximally projected images or using $3 \mathrm{D}$ coordinates. Three replicates are shown; each density curve represents at least 1000 distances. (B) Spearman's correlation between the same minimal spot-to-spot distance measured in $2 \mathrm{D}$ versus $3 \mathrm{D}$. 
previously shown to change the radial distribution of $L A D F$ and COL1A1 (Shachar et al. 2015). The analysis shows that the distribution of distances between $L A D F$ and $C O L 1 A 1$ is similar when analyzed in $2 \mathrm{D}$ versus $3 \mathrm{D}$ (Fig. 2A). Moreover, when the distance between each pair of spots in 2D was compared with the distance between the same pair in 3D the correlation was very high (Spearman's $\rho=0.998$ ) over 7000 measured distances (Fig. 2B). We conclude that spot-to-spot distance $2 \mathrm{D}$ analysis of projections is equivalent to $3 \mathrm{D}$ analysis.

\section{Versatility}

To date, HIPMap has been successfully applied to a variety of loci and cell types such as IMR90, MRC5, PC3, and HeLa, as well as lymphocytic cell lines such as ALCL (anaplastic large cell lymphoma) and Jurkat, and to hESCs (human embryonic stem cells). Adherent cells produce better results as a larger number of wash steps during the hiFISH protocol can be applied with minimal loss of cells. The endogenous loci that have been detected by HIPMap include active genes as well as highly repetitive gene deserts (see Fig. 5; Shachar et al. 2015). FISH probe design is limited only by the sequence covered by the specific BAC.

\section{QUALITY CONTROL PARAMETERS FOR HIPMap}

HIPMap is a robust and highly reproducible method as judged by several quality-control parameters.

\section{False FISH Signal Detection Rate}

Because all image analysis steps are fully automated, accurate detection of FISH signals is imperative. To determine the false detection rates of FISH signals, 15 image fields from five hiFISH experiments each containing approximately 300 cells were randomly chosen and the FISH spots identified either visually or by automated image analysis. Comparison of the data sets showed that the false-negative and -positive rates ranged between $0 \%$ and $9 \%$ for the three channels (Fig. 3A). False signal detection was due to real FISH signals not being detected by the software and fluorescent noise that is identified as signals. Additionally, when the number of FISH spots per cell was analyzed, at least $70 \%$ of cells contained two FISH spots as expected for the diploid CRL-1474 human skin fibroblasts used in the analysis (Fig. 3B).

\section{Determination of Minimal Sample Size}

HIPMap relies on the statistical analysis of a large number of data points per sample. Although HTI is capable of acquiring a very large number of cells, imaging time becomes a rate-limiting step in large-scale applications. The minimal number of FISH spots required to ensure high statistical fidelity was determined to be approximately 600 FISH spots using a computational simulation (Shachar et al. 2015). Briefly, 9000 FISH spots from nine different wells were pooled and two groups of equal numbers of spots were randomly chosen from this pool. Each two groups of spots were compared to one another using the KS test, and the simulation was repeated 10,000 times for varying spot numbers ranging from 100 to 2000 spots. Using a similar simulation, it is evident that distance distributions of 400 FISH spots or less do not faithfully capture the actual distribution of the population (Fig. 4A; $P>0.05)$. Distributions generated from less than 400 FISH spots are generally noisy and could be misleading when compared with other distributions generated from low cell numbers.

\section{Robustness of HIPMap Measurements}

Robustness of a method is defined as the degree of similarity among results generated from distinct subpopulations of a data set. HIPMap is highly robust as indicated by the fact that distributions generated from distinct groups of FISH signals randomly sampled from the same well were statistically indistinguishable $(P>0.1)$ (Fig. 4B). In addition, well-to-well and plate-to-plate variability is minimal $(P>0.1)$ (Fig. 4B).

\section{MAPPING OF SPATIAL GENE POSITIONS BY HIPMap}

\section{Determination of Gene Positions}

Representative data sets of HIPMap analysis of five diverse loci in hTERT (human telomerase reverse transcriptase)-immortalized CRL-1474 human skin fibroblasts are shown in Figure 5. The five loci differ in their chromatin environment, expression levels, and chromosomal location. $L A D F$ is a heterochromatic, nonexpressed gene desert located on chromosome 5 previously shown to associate with the nuclear lamina; $A C T B$ and $C O L 1 A 1$ represent active, euchromatic genes and reside on chromosomes 7 and 17, respectively. MMP9 and VEGF are both expressed at low levels in these cells and reside on chromosomes 20 and 6, respectively (Fig. 5). Mapping by HIPMap of these loci identified their distinct radial positions. As expected, most $\angle A D F$ signals are very close to the nuclear periphery with a median radial position of 0.2 (Fig. 5). COL1A1, on the other hand, has a much more internal distribution with a median radial position of 0.53 .

\section{Detection of Changes in Gene Positions}

A major question in the field concerns the mechanisms that determine the spatial location of gene loci in $3 \mathrm{D}$ space. Disruption of candidate mechanisms using RNAi is a possible approach to identify positioning mechanisms. To test whether HIPMap is sensitive enough to detect a change in the position of a gene, the effect of siRNA against $\operatorname{Lamin} A / C$ and $\operatorname{Lamin} B 1$, the major components of the nuclear lamina, on the location of the peripheral $L A D F$ locus was tested. Simultaneous knockdown of these genes by $>90 \%$ of their mRNA levels caused a detectable shift in the distribution of $L A D F$ 
A
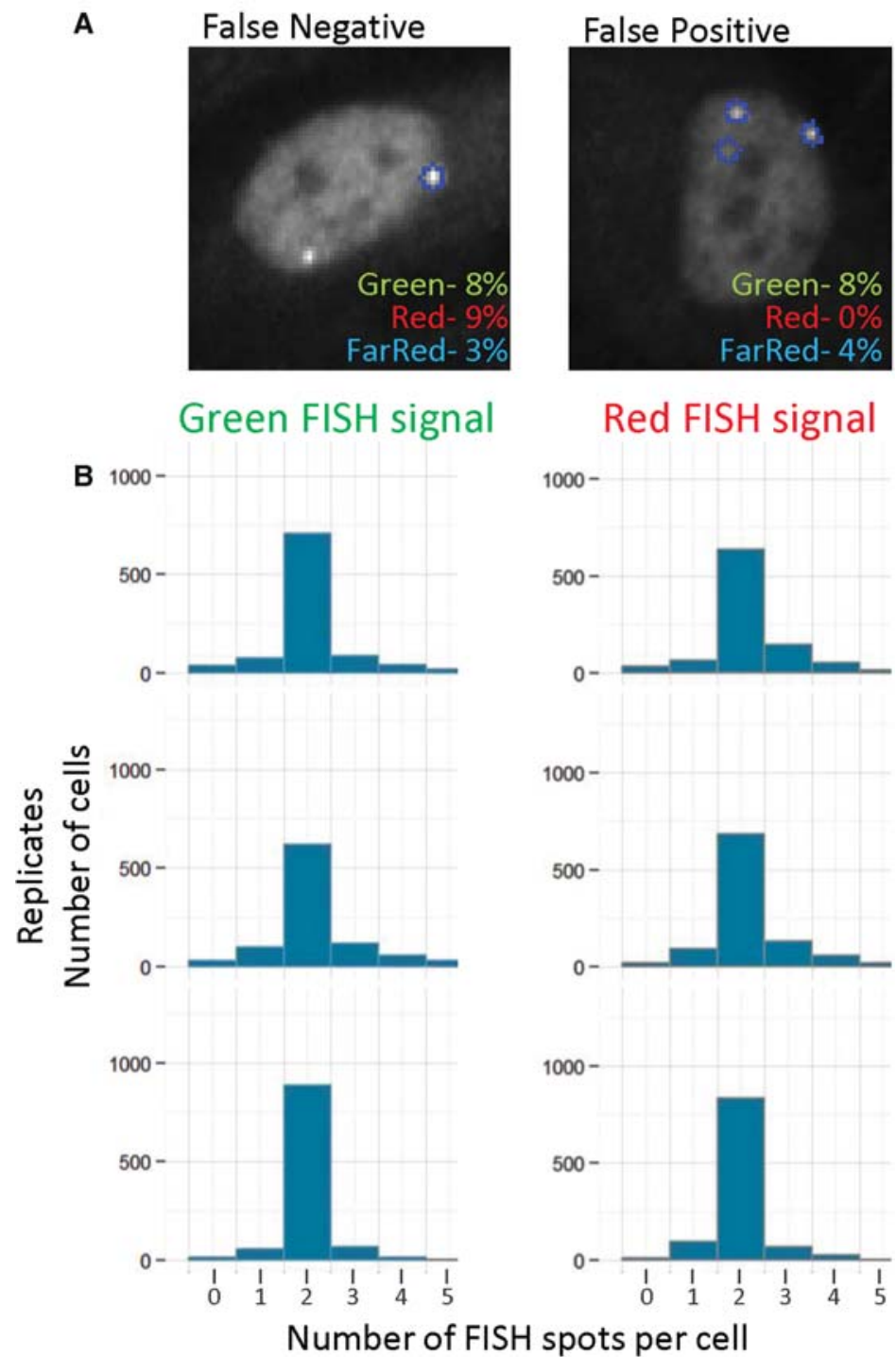

Figure 3. Accuracy of HIPMap image analysis. $(A)$ Representative images of maximally projected $z$-stacks. The false FISH spot detection rate was determined by cross comparison of automatically determined spots to manual detection for more than 300 cells in 15 randomly sampled fields. $(B)$ Number of FISH spots detected by image analysis software per cell, calculated for at least 700 cells per well. Replicate wells are shown for green $(488-\mathrm{nm})$ and red $(561-\mathrm{nm})$ channels.

(Fig. 6). The median distance from the periphery for the $L A D F$ locus changed from 0.2 in cells treated with a nontargeting siRNA to 0.29 in knockdown cells $(P<1 \times$ $\left.10^{-16}\right)$. Similar effects of lamin loss were found for three other genes in these cells (Shachar et al. 2015).

\section{APPLICATIONS OF HIPMap}

HIPMap is a versatile assay that can be used to answer several basic biological questions relevant to chromatin organization as well as more translational questions.

\section{Scaling-Up of HIPMap}

A major potential application is the combination of HIPMap with in silico design and synthetic synthesis of
FISH oligo probes enabling the scaling-up of gene mapping and screens to probe the location of large sets of genomic targets in a single experiment (Beliveau et al. 2012; Joyce et al. 2012). Large-scale probe design methods, such as Oligopaints, are now available and can be combined with HIPMap. In Oligopaints and its variants (Beliveau et al. 2012, 2014, 2015), large libraries of DNA oligos tiling genomic regions of interest are designed in silico and chemically synthetized. These libraries of DNA oligos can be either directly labeled with fluorophores or designed to include de novo DNA barcodes that can be used as hybridization platforms for fluorescent signal amplification or for multiplexing the number of nucleic acid targets detected in a single sample with imaging approaches (Beliveau et al. 2015; Chen et al. 2015). An obvious obstacle to large-scale FISH experiments is 
A

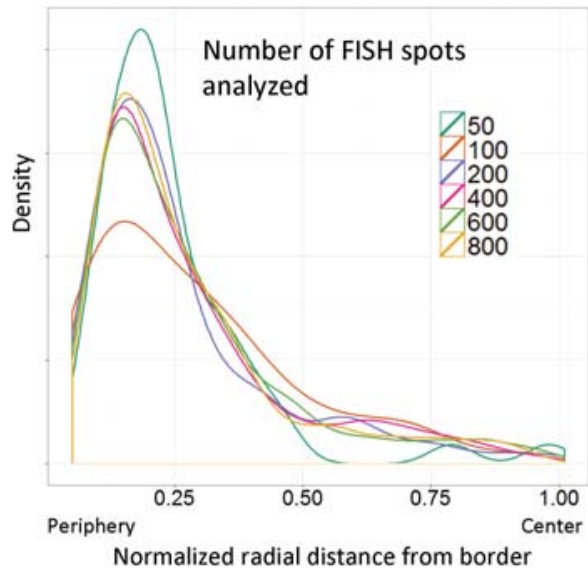

B

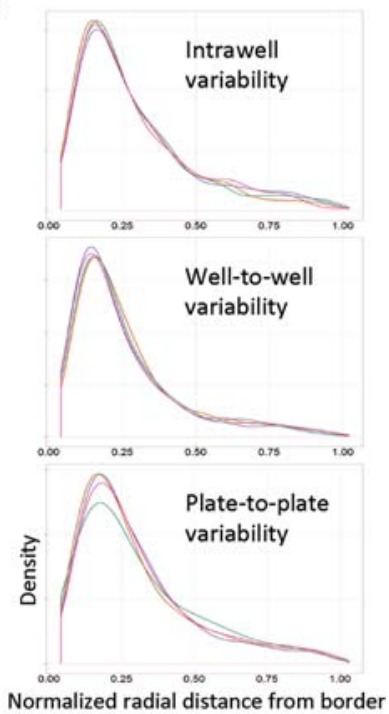

Figure 4. Robustness and reproducibility of HIPMap. (A) Subsampling of data sets demonstrates that radial distance distributions generated from less than 400 FISH spots do not faithfully represent real distribution. Density curves are generated from the indicated number of FISH spots randomly extracted from a pool of 9000 spots from nine replicate wells. $(B)$ Estimated density curves of radial distances generated from more than 600 FISH spots per replicate from the same well, replicate wells on the same plate, or wells in different plates.

the resulting long imaging times. For example, screening of an siRNA library of 700 candidate genes testing three target loci required a total of 650 imaging hours (Shachar et al. 2015). New generations of high-throughput microscopes with larger camera chips enabling the acquisition of a larger number of cells per field of view and more powerful light sources allowing shorter exposure times will likely overcome this issue in the near future.

\section{RNAi and Small Molecule Screens}

We have recently used HIPMap to carry out an siRNA screen to discover factors that contribute to determining the position of four functionally diverse genes in human skin fibroblasts (Fig. 7; Shachar et al. 2015). Using an siRNA library targeting approximately 700 genes encoding nuclear proteins, 2036 wells were imaged and more than three million data points analyzed. The screen identified 50 positioning factors representing a first compendium of cellular proteins that determine the spatial location of human genes (Shachar et al. 2015).

Similar screens can be envisioned in any desired adherent cell type and using diverse set of libraries. For example, it would be interesting to explore the effect of interference with cellular signaling pathways on gene positioning. HIPMap is potentially equally suited for screening of small molecule libraries. Finally, it is foreseeable to apply HIPMap in combination with CRISPR-based approaches to either knockout or overexpress genes on a large scale.

\section{Systematic Mapping of 3D Distances}

HIPMap is ideally suited to measure distances between gene loci. The ability to generate large probe sets target- ing custom-defined regions of the genome opens the door to the systematic mapping of locus-to-locus 3D distances between thousands of genomic loci in different biological conditions or in different genetic backgrounds (Fig. 7). The capacity to systematically triangulate the distances of large sets of genome loci has the potential to generate low-resolution 3D genome maps.

\section{Validation of Chromosome Conformation Capture Data Sets}

HIPMap is envisioned to become a valuable orthogonal tool to validate chromosome conformation capture (3C) interaction data (Williamson et al. 2014). In one possible scenario, HIPMap could be used to measure 3D distance distributions between a constant genomic locus and a set of tiled FISH probes designed to hybridize at loci positioned at increasing distances away from the bait locus. In addition, Hi-C interactors of similar signal strength, but varying genomic distances, should be systematically analyzed to determine how signal strength and interaction frequencies are related. Alternatively, a large number of loops predicted by high-resolution Hi$\mathrm{C}$ mapping could be analyzed to determine the percentage of cells that show contacts between genomic regions at the base of the loop. Similarly, interchromosomal interactions can be systematically tested for their prevalence in the cell population.

\section{Determination of Chromosome Breaks and Translocations}

Given its capability to measure relational spatial distances of up to three independently labeled genomic 


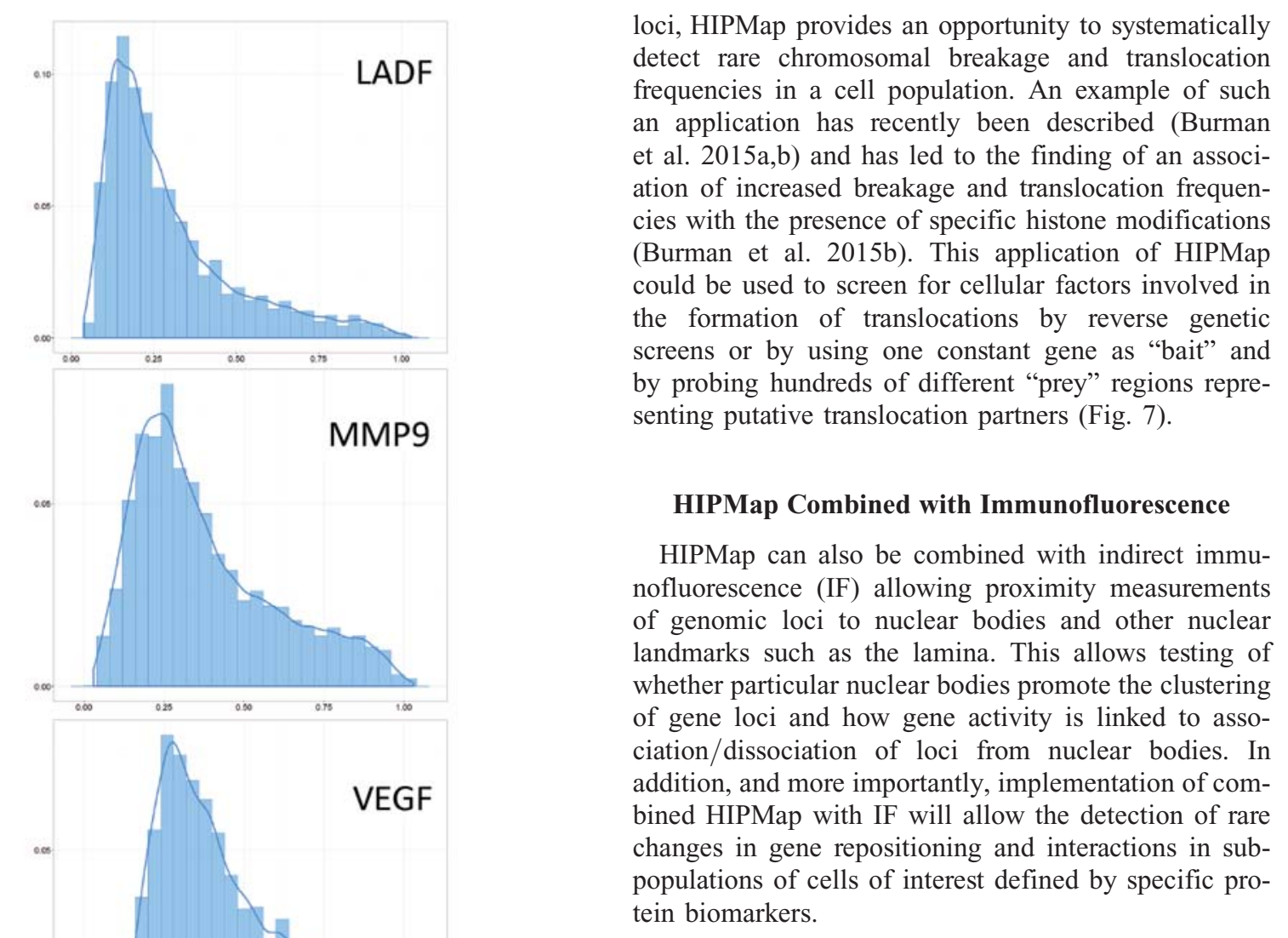

Figure 5. Distributions of endogenous loci. Radial distance distributions (bars) and estimated density curves (lines) of the indicated gene locus. Distributions are generated from more than 600 FISH spots per locus.
A

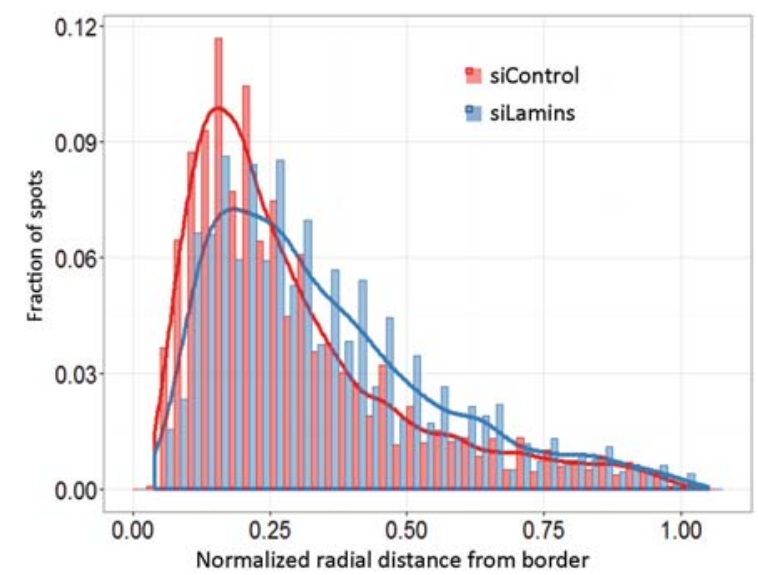

B

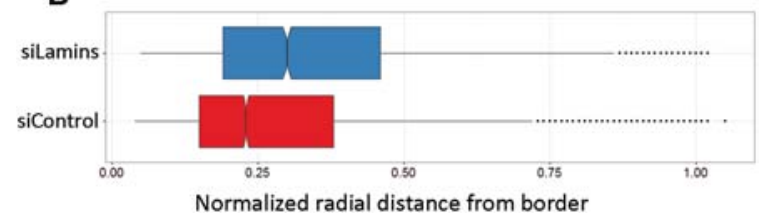

Figure 6. HIPMap detects changes in positioning. (A) Radial distance distributions following siRNA transfection for the indicated gene of the $L A D F$ locus. $(B)$ Boxplot of radial distances of the $L A D F$ locus to the nucleus border in cells treated with the indicated siRNA for $72 \mathrm{~h}$. Boxes are 25th, 50th (median), and 75 th percentile of the distributions and whiskers extend up to 1.5 interquantile range; outliers are shown as dots.

loci, HIPMap provides an opportunity to systematically frequencies in a cell population. An example of such et al. 2015a,b) and has led to the finding of an associcies with the presence of specific histone modifications (Burman et al. 2015b). This application or HIPMap the formation of translocations by reverse genetic by probing hundreds of different "prey" regions repre-

HIPMap can also be combined with indirect immuof genomic loci to nuclear bodies and other nuclear whether particular nuclear bodies promote the clustering of gene loci and how gene activity is linked to assoaddition, and more importantly, implementation of comchanges in gene repositioning and interactions in subtein biomarkers. 


\begin{tabular}{|c|c|c|}
\hline Application & Assay & Potential insights \\
\hline Screens & $\begin{array}{l}\text { hiFISH of } \\
\text { multiple } \\
\text { endogenous loci }\end{array}$ & $\begin{array}{l}\text { - Identification of genome organization } \\
\text { factors } \\
\text { - Association between expression and } \\
\text { positioning }\end{array}$ \\
\hline $\begin{array}{l}\text { Breakage and translocation } \\
\text { mapping }\end{array}$ & $\begin{array}{l}\text { hiFISH using } \\
\text { break apart } \\
\text { probes }\end{array}$ & $\begin{array}{l}\text { - Effect of chromatin modifiers on } \\
\text { breakage susceptibility } \\
\text { - Dynamics of breakage/repair in } \\
\text { different cell types }\end{array}$ \\
\hline $\mathrm{Hi}-\mathrm{C}$ data validation & $\begin{array}{l}\text { hiFISH of } \\
\text { predicted } \\
\text { interactors }\end{array}$ & $\begin{array}{l}\text { - Relationship between } \mathrm{Hi}-\mathrm{C} \text { contact } \\
\text { maps and physical interactions in } \\
\text { single cells }\end{array}$ \\
\hline Interactions with nuclear landmarks & $\begin{array}{l}\text { hiFISH combined } \\
\text { with IF of nuclear } \\
\text { proteins }\end{array}$ & $\begin{array}{l}\text { - Effect of nuclear factors on association } \\
\text { of genome regions with nuclear } \\
\text { bodies and structures }\end{array}$ \\
\hline Functional/ mec & $\begin{array}{l}\text { hiFISH combined } \\
\text { with IF of cellular } \\
\text { markers / RNA } \\
\text { FISH }\end{array}$ & $\begin{array}{l}\text { - Relationship of gene expression with } \\
\text { nuclear positioning and vice versa } \\
\text { - Difference in positioning in sub- } \\
\text { populations of cells } \\
\text { - Changes in positioning in different } \\
\text { developmental stage, cell types. }\end{array}$ \\
\hline
\end{tabular}

Figure 7. Applications of HIPMap.

\section{HIPMap-RNA FISH}

Finally, coupling HIPMap with RNA FISH provides a single-allele, high-throughput view of the relationships between genome positioning and transcriptional activity. This approach will be particularly important to test on a large scale whether known changes in gene expression associated with physiological changes are accompanied by changes in radial position of the corresponding genomic locus (Therizols et al. 2014).

\section{CONCLUSION}

The HIPMap pipeline is an optimized tool to map the spatial positions of genes in the intact cell nucleus. The availability of a method that combines the power of direct visualization of gene loci and single-cell imaging with the ability to interrogate genomes at a large-scale overcomes some of the limitations inherent to the two major methods, microscopy and 3C technology, that have been used to probe higher-order genome organization. HIPMap is a highly modular technology, and combination of HIPMap with the development of more complex probe libraries, with other detection methods, and with the use of genetic tools will further enhance the impact of the method. Regardless of the nature of future applications, HIPMap has the potential to contribute significantly to the elucidation of genome structure and function.

\section{ACKNOWLEDGMENTS}

We thank Karen Meaburn for help with developing hiFISH and members of the Misteli laboratory for helpful discussions and comments. This research was supported by the Intramural Research Program of the National Institutes of Health (NIH), the National Cancer Institute (NCI), and the Center for Cancer Research.

\section{REFERENCES}

Beliveau BJ, Joyce EF, Apostolopoulos N, Yilmaz F, Fonseka CY, McCole RB, Chang Y, Li JB, Senaratne TN, Williams BR, et al. 2012. Versatile design and synthesis platform for visualizing genomes with Oligopaint FISH probes. Proc Natl Acad Sci 109: 21301-21306.

Beliveau BJ, Apostolopoulos N, Wu CT. 2014. Visualizing genomes with Oligopaint FISH probes. Curr Protoc Mol Biol 105: Unit 1423.

Beliveau BJ, Boettiger AN, Avendaño MS, Jungmann R, McCole RB, Joyce EF, Kim-Kiselak C, Bantignies F, Fonseka CY, Erceg J, et al. 2015. Single-molecule super-resolution imaging of chromosomes and in situ haplotype visualization using Oligopaint FISH probes. Nat Commun 6: 7147. 
Burman B, Misteli T, Pegoraro G. 2015a. Quantitative detection of rare interphase chromosome breaks and translocations by high-throughput imaging. Genome Biol 16: 146.

Burman B, Zhang ZZ, Pegoraro G, Lieb JD, Misteli T. 2015b. Histone modifications predispose genome regions to breakage and translocation. Genes Dev 29: 1393-1402.

Cavalli G, Misteli T. 2013. Functional implications of genome topology. Nat Struct Mol Biol 20: 290-299.

Chen KH, Boettiger AN, Moffitt JR, Wang S, Zhuang X. 2015. RNA imaging. Spatially resolved, highly multiplexed RNA profiling in single cells. Science 348: aaa6090.

Conrad C, Gerlich DW. 2010. Automated microscopy for highcontent RNAi screening. J Cell Biol 188: 453-461.

Cremer T, Cremer M. 2010. Chromosome territories. Cold Spring Harb Perspect Biol 2: a003889.

Dekker J, Misteli T. 2015. Long-range chromatin interactions. In Epigenetics, 2nd ed. (ed. Allis CD, et al.), pp. 507-528. Cold Spring Harbor Laboratory Press, Cold Spring Harbor, NY

Dekker J, Marti-Renom MA, Mirny LA. 2013. Exploring the three-dimensional organization of genomes: Interpreting chromatin interaction data. Nat Rev Genet 14: 390-403.

de Wit E, de Laat W. 2012. A decade of 3C technologies: Insights into nuclear organization. Genes Dev 26: 11-24.

Dixon JR, Selvaraj S, Yue F, Kim A, Li Y, Shen Y, Hu M, Liu JS, Ren B. 2012. Topological domains in mammalian genomes identified by analysis of chromatin interactions. Nature $\mathbf{4 8 5}$ 376-380.

Joyce EF, Williams BR, Xie T, Wu CT. 2012. Identification of genes that promote or antagonize somatic homolog pairing using a high-throughput FISH-based screen. PLoS Genet 8: e1002667.

Meaburn KJ, Misteli T. 2008. Locus-specific and activity-independent gene repositioning during early tumorigenesis. $J$ Cell Biol 180: $39-50$

Meaburn KJ, Gudla PR, Khan S, Lockett SJ, Misteli T. 2009. Disease-specific gene repositioning in breast cancer. $J$ Cell Biol 187: 801-812.
Misteli T. 2007. Beyond the sequence: Cellular organization of genome function. Cell 128: 787-800.

Misteli T. 2013. The cell biology of genomes: Bringing the double helix to life. Cell 152: 1209-1212.

Mitchison TJ. 2005. Small-molecule screening and profiling by using automated microscopy. Chembiochem 6: 33-39.

Nagano T, Lubling Y, Stevens TJ, Schoenfelder S, Yaffe E, Dean W, Laue ED, Tanay A, Fraser P. 2013. Single-cell Hi$\mathrm{C}$ reveals cell-to-cell variability in chromosome structure. Nature 502: 59-64.

Perlman ZE, Slack MD, Feng Y, Mitchison TJ, Wu LF, Altschuler SJ. 2004. Multidimensional drug profiling by automated microscopy. Science 306: 1194-1198.

Roukos V, Misteli T, Schmidt CK. 2010. Descriptive no more: The dawn of high-throughput microscopy. Trends Cell Biol 20: $503-506$.

Roukos V, Voss TC, Schmidt CK, Lee S, Wangsa D, Misteli T. 2013. Spatial dynamics of chromosome translocations in living cells. Science 341: 660-664.

Sexton T, Yaffe E, Kenigsberg E, Bantignies F, Leblanc B, Hoichman M, Parrinello H, Tanay A, Cavalli G. 2012. Three-dimensional folding and functional organization principles of the Drosophila genome. Cell 148: 458-472.

Shachar S, Voss TC, Pegoraro G, Sciascia N, Misteli T. 2015. Identification of gene positioning factors using high-throughput imaging mapping. Cell 162: 911-923.

Smellie A, Wilson CJ, Ng SC. 2006. Visualization and interpretation of high content screening data. J Chem Inf Model 46: 201-207.

Therizols P, Illingworth RS, Courilleau C, Boyle S, Wood AJ, Bickmore WA. 2014. Chromatin decondensation is sufficient to alter nuclear organization in embryonic stem cells. Science 346: $1238-1242$.

Williamson I, Berlivet S, Eskeland R, Boyle S, Illingworth RS, Paquette D, Dostie J, Bickmore WA. 2014. Spatial genome organization: Contrasting views from chromosome conformation capture and fluorescence in situ hybridization. Genes Dev 28: $2778-2791$. 


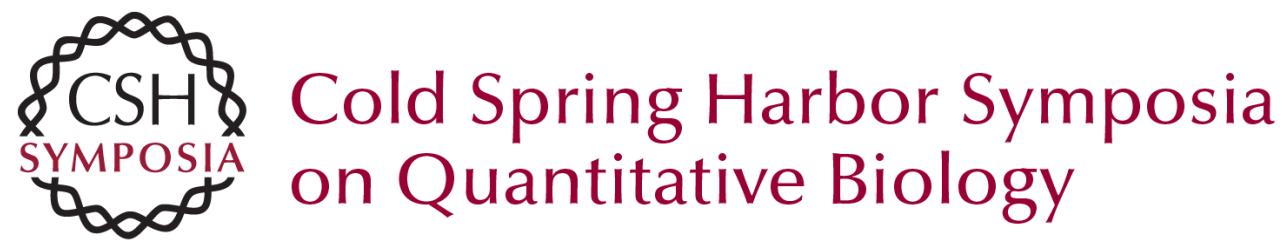

\section{HIPMap: A High-Throughput Imaging Method for Mapping Spatial Gene Positions}

Sigal Shachar, Gianluca Pegoraro and Tom Misteli

Cold Spring Harb Symp Quant Biol 2015 80: 73-81 originally published online October 15, 2015 Access the most recent version at doi:10.1101/sqb.2015.80.027417

References This article cites 26 articles, 12 of which can be accessed free at: http://symposium.cshlp.org/content/80/73.full.html\#ref-list-1

License

Email Alerting Receive free email alerts when new articles cite this article - sign up in Service the box at the top right corner of the article or click here. 